\title{
CONTACT PAIR STRUCTURES AND ASSOCIATED METRICS
}

\author{
G. BANDE AND A. HADJAR
}

\begin{abstract}
We introduce the notion of contact pair structure and the corresponding associated metrics, in the same spirit of the geometry of almost contact structures. We prove that, with respect to these metrics, the integral curves of the Reeb vector fields are geodesics and that the leaves of the Reeb action are totally geodesic. Moreover, we show that, in the case of a metric contact pair with decomposable endomorphism, the characteristic foliations are orthogonal and their leaves carry induced contact metric structures.
\end{abstract}

\section{INTRODUCTION}

A contact pair on a smooth manifold $M$ is a pair of one-forms $\alpha_{1}$ and $\alpha_{2}$ of constant and complementary classes, for which $\alpha_{1}$ restricted to the leaves of the characteristic foliation of $\alpha_{2}$ is a contact form and vice versa. This definition was introduced in [1, 3] and is the analogous to that of contact-symplectic pairs and symplectic pairs (see [2, 7, 10]).

In this paper we introduce the notion of contact pair structure, that is a contact pair on a manifold $M$ endowed with a tensor field $\phi$, of type $(1,1)$, such that $\phi^{2}=-I d+\alpha_{1} \otimes Z_{1}+\alpha_{2} \otimes Z_{2}$ and $\phi\left(Z_{1}\right)=\phi\left(Z_{2}\right)=0$, where $Z_{1}$ and $Z_{2}$ are the Reeb vector fields of the pair.

We define compatible and associated metrics and we prove several properties of these metrics. For example, we show that the orbits of the locally free $\mathbb{R}^{2}$-action generated by the two commuting Reeb vector fields are totally geodesic with respect to these metrics. Another important feature is that, given a metric which is associated with respect to a contact pair structure $\left(\alpha_{1}, \alpha_{2}, \phi\right)$, if we assume that the endomorphism $\phi$ preserves the tangent spaces of the leaves of the two characteristic foliations, then the contact forms induced on the leaves are contact metric structures.

In what follows we denote by $\Gamma(B)$ the space of sections of a vector bundle $B$. For a given foliation $\mathcal{F}$ on a manifold $M$, we denote by $T \mathcal{F}$ the subbundle of $T M$ whose fibers are given by the distribution tangent to the leaves. All the differential objects considered are supposed smooth.

\section{PRELIMINARIES ON CONTACT PAIRS}

We gather in this section the notions concerning contact pairs that will be needed in the sequel. We refer the reader to [2, 3, 6, 7] for further informations and several examples of such structures.

Definition 1. A pair $\left(\alpha_{1}, \alpha_{2}\right)$ of 1 -forms on a manifold is said to be a contact pair of type $(h, k)$ if:

i) $\alpha_{1} \wedge\left(d \alpha_{1}\right)^{h} \wedge \alpha_{2} \wedge\left(d \alpha_{2}\right)^{k}$ is a volume form,

ii) $\left(d \alpha_{1}\right)^{h+1}=0$,

iii) $\left(d \alpha_{2}\right)^{k+1}=0$.

2000 Mathematics Subject Classification. Primary 53C15; Secondary 53D15, 53C12, 53D35.

Key words and phrases. contact pairs, foliation, associated metrics, contact metric structure.

The first author was supported by the MIUR Project: Riemannian metrics and differentiable manifolds-P.R.I.N. 2005 . 
Since the form $\alpha_{1}$ (resp. $\alpha_{2}$ ) has constant class $2 h+1$ (resp. $2 k+1$ ), the distribution Ker $\alpha_{1} \cap$ Ker $d \alpha_{1}$ (resp. Ker $\alpha_{2} \cap \operatorname{Ker} d \alpha_{2}$ ) is completely integrable and then it determines the so-called characteristic foliation $\mathcal{F}_{1}$ (resp. $\mathcal{F}_{2}$ ) whose leaves are endowed with a contact form induced by $\alpha_{2}$ (resp. $\left.\alpha_{1}\right)$.

To a contact pair $\left(\alpha_{1}, \alpha_{2}\right)$ of type $(h, k)$ are associated two commuting vector fields $Z_{1}$ and $Z_{2}$, called Reeb vector fields of the pair, which are uniquely determined by the following equations:

$$
\begin{gathered}
\alpha_{1}\left(Z_{1}\right)=\alpha_{2}\left(Z_{2}\right)=1, \quad \alpha_{1}\left(Z_{2}\right)=\alpha_{2}\left(Z_{1}\right)=0, \\
i_{Z_{1}} d \alpha_{1}=i_{Z_{1}} d \alpha_{2}=i_{Z_{2}} d \alpha_{1}=i_{Z_{2}} d \alpha_{2}=0,
\end{gathered}
$$

where $i_{X}$ is the contraction with the vector field $X$. In particular, since the Reeb vector fields commute, they determine a locally free $\mathbb{R}^{2}$-action, called the Reeb action.

The kernel distribution of $d \alpha_{1}$ (resp. $d \alpha_{2}$ ) is also integrable and then it defines a foliation whose leaves inherit a contact pair of type $(0, k)($ resp. $(h, 0))$.

A contact pair of type $(h, k)$ has a local model (see [3, 2]), which means that for every point of the manifold there exists a coordinate chart on which the pair can be written as follows:

$$
\alpha_{1}=d x_{2 h+1}+\sum_{i=1}^{h} x_{2 i-1} d x_{2 i}, \quad \alpha_{2}=d y_{2 k+1}+\sum_{i=1}^{k} y_{2 i-1} d y_{2 i}
$$

where $\left(x_{1}, \cdots, x_{2 h+1}, y_{1}, \cdots, y_{2 k+1}\right)$ are the standard coordinates on $\mathbb{R}^{2 h+2 k+2}$.

The tangent bundle of a manifold $M$ endowed with a contact pair $\left(\alpha_{1}, \alpha_{2}\right)$ can be split in different ways. For $i=1,2$, let $T \mathcal{F}_{i}$ be the subbundle determined by the characteristic foliation of $\alpha_{i}, T \mathcal{G}_{i}$ the subbundle of $T M$ whose fibers are given by ker $d \alpha_{i} \cap \operatorname{ker} \alpha_{1} \cap \operatorname{ker} \alpha_{2}$ and $\mathbb{R} Z_{1}, \mathbb{R} Z_{2}$ the line bundles determined by the Reeb vector fields. Then we have the following splittings:

$$
T M=T \mathcal{F}_{1} \oplus T \mathcal{F}_{2}=T \mathcal{G}_{1} \oplus T \mathcal{G}_{2} \oplus \mathbb{R} Z_{1} \oplus \mathbb{R} Z_{2} .
$$

Moreover we have $T \mathcal{F}_{1}=T \mathcal{G}_{1} \oplus \mathbb{R} Z_{2}$ and $T \mathcal{F}_{2}=T \mathcal{G}_{2} \oplus \mathbb{R} Z_{1}$.

\section{CONTACT PAIR STRUCTURES AND ALMOST CONTACT STRUCTURES}

In this section we firstly recall the basic definitions of almost contact structures and their associated metrics. Next we introduce a new structure, namely the contact pair structure. More details on almost contact structures can be found in [8].

3.1. Almost contact structures. An almost contact structure on a manifold $M$ is a triple $(\alpha, Z, \phi)$ of a one-form $\alpha$, a vector field $Z$ and a field of endomorphisms $\phi$ of the tangent bundle of $M$, such that $\phi^{2}=-I d+\alpha \otimes Z, \phi(Z)=0$ and $\alpha(Z)=1$. In particular, it follows that $\alpha \circ \phi=0$ and that the rank of $\phi$ is $\operatorname{dim} M-1$.

In the study of almost contact structures, there are two types of metrics which are particularly interesting and we recall their definition:

Definition 2. For a given almost contact structure $(\alpha, Z, \phi)$ on a manifold $M$, a Riemannian metric $g$ is called:

i) compatible if $g(\phi X, \phi Y)=g(X, Y)-\alpha(X) \alpha(Y)$ for every $X, Y \in \Gamma(T M)$;

ii) associated if $g(X, \phi Y)=d \alpha(X, Y)$ and $g(X, Z)=\alpha(X)$ for every $X, Y \in \Gamma(T M)$. 
In particular if $g$ is an associated metric with respect to $(\alpha, Z, \phi)$, then $\alpha$ must be a contact form. For a given almost contact structure there always exists a compatible metric and for a given contact form there always exists an associated metric.

Definition 3. A contact metric structure $(\alpha, Z, \phi, g)$ is an almost contact structure $(\alpha, Z$, $\phi)$, where $\alpha$ is a contact form and $Z$ its Reeb vector field, together with an associated metric $g$.

3.2. Contact pair structures. Now we want to generalize the notion of contact metric structure to the contact pairs. To do that, we begin with the following definition:

Definition 4. A contact pair structure on a manifold $M$ is a triple $\left(\alpha_{1}, \alpha_{2}, \phi\right)$, were $\left(\alpha_{1}, \alpha_{2}\right)$ is a contact pair and $\phi$ a tensor field of type $(1,1)$ such that:

$$
\begin{gathered}
\phi^{2}=-I d+\alpha_{1} \otimes Z_{1}+\alpha_{2} \otimes Z_{2}, \\
\phi\left(Z_{1}\right)=\phi\left(Z_{2}\right)=0 .
\end{gathered}
$$

where $Z_{1}$ and $Z_{2}$ are the Reeb vector fields of $\left(\alpha_{1}, \alpha_{2}\right)$.

As in the case of an almost contact structure, it is easy to check that $\alpha_{i} \circ \phi=0, i=1,2$ and the rank of $\phi$ is equal to $\operatorname{dim} M-2$.

Remark 5. Actually, the condition $\phi(Z)=0$ in the definition of almost contact structure is not needed, since it follows from $\phi^{2}=-I+\alpha \otimes Z$ (see [8], Theorem $4.1 \mathrm{p}$. 33). In the case of a contact pair, it is possible to construct an endomorphism satisfying (1) but not (2). This can be done, for example, by taking $\phi$ to be an almost complex structure on $T \mathcal{G}_{1} \oplus T \mathcal{G}_{2}$ and extending it by setting $\phi\left(Z_{1}\right)=-\phi\left(Z_{2}\right)=Z_{1}+Z_{2}$.

On a manifold $M$ endowed with a contact pair, there always exists an endomorphism $\phi$ verifying (1) and (2), since on the subbundle $T \mathcal{G}_{1} \oplus T \mathcal{G}_{2}$ of $T M$, the 2 -form $d \alpha_{1}+d \alpha_{2}$ is symplectic. Then one can choose an almost complex structure on $T \mathcal{G}_{1} \oplus T \mathcal{G}_{2}$ and extend it to be zero on the Reeb vector fields, to produce an endomorphism $\phi$ of $T M$ with the desired properties.

The following definition is justified by the fact that we are interested on the contact structures induced on the leaves of the characteristic foliations of a contact pair.

Definition 6. The endomorphism $\phi$ is said to be decomposable if $\phi\left(T \mathcal{F}_{i}\right) \subset T \mathcal{F}_{i}$, for $i=1,2$.

The condition for $\phi$ to be decomposable is equivalent to $\phi\left(T \mathcal{G}_{i}\right)=T \mathcal{G}_{i}$, because $\phi$ vanishes on $Z_{1}, Z_{2}$ and has rank $2 k$ (resp. $2 h$ ) on $T \mathcal{F}_{1}$ (resp. $T \mathcal{F}_{2}$ ).

By Definition 1, $\alpha_{1}$ induces a contact form on the leaves of the characteristic foliation of $\alpha_{2}$ and vice versa. Then it is easy to prove the following:

Proposition 7. If $\phi$ is decomposable, then $\left(\alpha_{1}, Z_{1}, \phi\right)$ (resp. $\left.\left(\alpha_{2}, Z_{2}, \phi\right)\right)$ induces an almost contact structure on the leaves of $\mathcal{F}_{2}$ (resp. $\left.\mathcal{F}_{1}\right)$.

Here is a simple example of contact pair on a manifold which is just a product of two contact manifolds and $\phi$ is not decomposable:

Example 8. Let us consider standard coordinates $\left(x_{1}, y_{1}, x_{2}, y_{2}, z_{1}, z_{2}\right)$ on $\mathbb{R}^{6}$ and the contact pair $\left(\alpha_{1}, \alpha_{2}\right)$ given by:

$$
\alpha_{1}=d z_{1}-x_{1} d y_{1} \quad \alpha_{2}=d z_{2}-x_{2} d y_{2}
$$


with Reeb vector fields $Z_{1}=\frac{\partial}{\partial z_{1}}, Z_{2}=\frac{\partial}{\partial z_{2}}$. Let us define $\phi$ as follows:

$$
\begin{gathered}
\phi\left(a_{1} \frac{\partial}{\partial x_{1}}+b_{1} \frac{\partial}{\partial y_{1}}+a_{2} \frac{\partial}{\partial x_{2}}+b_{2} \frac{\partial}{\partial y_{2}}+c_{1} \frac{\partial}{\partial z_{1}}+c_{2} \frac{\partial}{\partial z_{2}}\right)= \\
=\left(-a_{2} \frac{\partial}{\partial x_{1}}-b_{2} \frac{\partial}{\partial y_{1}}+a_{1} \frac{\partial}{\partial x_{2}}+b_{1} \frac{\partial}{\partial y_{2}}-x_{1} b_{2} \frac{\partial}{\partial z_{1}}+x_{2} b_{1} \frac{\partial}{\partial z_{2}}\right),
\end{gathered}
$$

where $a_{1}, a_{2}, b_{1}, b_{2}, c_{1}, c_{2}$ are smooth functions on $\mathbb{R}^{6}$.

Then $\left(\alpha_{1}, \alpha_{2}, \phi\right)$ is a contact pair structure and $\phi$ is not decomposable. This because, for example, $\frac{\partial}{\partial x_{1}} \in \operatorname{Ker}\left(\alpha_{2} \wedge d \alpha_{2}\right), \frac{\partial}{\partial x_{2}} \in \operatorname{Ker}\left(\alpha_{1} \wedge d \alpha_{1}\right)$ but $\phi\left(\frac{\partial}{\partial x_{1}}\right)=\frac{\partial}{\partial x_{2}}$.

Remark 9. A more general structure, similar to the almost contact structures, is obtained by considering a 5-tuple $\left(\alpha_{1}, \alpha_{2}, Z_{1}, Z_{2}, \phi\right)$, where $\phi^{2}=-I d+\alpha_{1} \otimes Z_{1}+\alpha_{2} \otimes Z_{2}, \phi\left(Z_{1}\right)=\phi\left(Z_{2}\right)=0$ and the $\alpha_{i}$ 's are just non-vanishing 1-forms verifying $\alpha_{i}\left(Z_{j}\right)=\delta_{i j}$.

\section{Compatible AND ASSOCIATED METRICS}

For a given contact pair structure on a manifold $M$, it is natural to consider the following metrics:

Definition 10. Let $\left(\alpha_{1}, \alpha_{2}, \phi\right)$ be a contact pair structure on a manifold $M$, with Reeb vector fields $Z_{1}$ and $Z_{2}$. A Riemannian metric $g$ on $M$ is called:

i) compatible if $g(\phi X, \phi Y)=g(X, Y)-\alpha_{1}(X) \alpha_{1}(Y)-\alpha_{2}(X) \alpha_{2}(Y)$ for all $X, Y \in \Gamma(T M)$,

ii) associated if $g(X, \phi Y)=\left(d \alpha_{1}+d \alpha_{2}\right)(X, Y)$ and $g\left(X, Z_{i}\right)=\alpha_{i}(X)$, for $i=1,2$ and for all $X, Y \in \Gamma(T M)$.

It is clear that an associated metric is also compatible, but the converse is not true. In the Example 8, the metric $g=\sum_{i=1}^{2}\left(d x_{i}^{2}+d y_{i}^{2}+\alpha_{i}^{2}\right)$ is compatible but not associated.

Definition 11. A metric contact pair (MCP) on a manifold $M$ is a 4-tuple $\left(\alpha_{1}, \alpha_{2}, \phi, g\right)$ where $\left(\alpha_{1}, \alpha_{2}, \phi\right)$ is a contact pair structure and $g$ an associated metric with respect to it.

Like for compatible metrics on almost contact manifolds, we have:

Proposition 12. For every contact pair structure on a manifold $M$ there exists a compatible metric.

Proof. Let $\left(\alpha_{1}, \alpha_{2}, \phi\right)$ be a contact pair structure on $M$. Pick any Riemannian metric $h$ on $M$ and define $k$ as

$$
k(X, Y)=h\left(\phi^{2} X, \phi^{2} Y\right)+\alpha_{1}(X) \alpha_{1}(Y)+\alpha_{2}(X) \alpha_{2}(Y) .
$$

Now put

$$
g(X, Y)=\frac{1}{2}\left(k(X, Y)+k(\phi X, \phi Y)+\alpha_{1}(X) \alpha_{1}(Y)+\alpha_{2}(X) \alpha_{2}(Y)\right) .
$$

It is straightforward to show that $g$ is a compatible metric with respect to $\left(\alpha_{1}, \alpha_{2}, \phi\right)$.

Compatible and associated metrics have several interesting properties given by the following

Theorem 13. Let $M$ be a manifold endowed with a contact pair structure $\left(\alpha_{1}, \alpha_{2}, \phi\right)$, with Reeb vector fields $Z_{1}, Z_{2}$. Let $g$ be a compatible metric with respect to it, with Levi-Cività connection $\nabla$. Then we have:

(1) $g\left(Z_{i}, X\right)=\alpha_{i}(X)$, for $i=1,2$ and for every $X \in \Gamma(T M)$;

(2) $g\left(Z_{i}, Z_{j}\right)=\delta_{i}^{j}, i, j=1,2$;

(3) $\nabla_{Z_{i}} Z_{j}=0, i, j=1,2$ (in particular the integral curves of the Reeb vector fields are geodesics); 
(4) the Reeb action is totally geodesic (i.e the orbits are totally geodesic 2-dimensional submanifolds).

Moreover, if $g$ is an associated metric and $L_{Z_{i}}$ is the Lie derivative along $Z_{i}$, then $L_{Z_{i}} \phi=0$ if and only if $Z_{i}$ is a Killing vector field.

Proof. The first two properties are easy consequences of the definitions of compatible metric and of the Reeb vector fields of a contact pair. For the third property, let us remember that $\alpha_{i}$ is invariant by $Z_{j}, i, j=1,2$, and then:

$$
\begin{aligned}
0 & =\left(L_{Z_{j}} \alpha_{i}\right)(X)=Z_{j}\left(\alpha_{i}(X)\right)-\alpha_{i}\left(L_{Z_{j}} X\right) \\
& =Z_{j}\left(g\left(X, Z_{i}\right)\right)-g\left(Z_{i}, \nabla_{Z_{j}} X-\nabla_{X} Z_{j}\right) \\
& =g\left(\nabla_{Z_{j}} X, Z_{i}\right)+g\left(X, \nabla_{Z_{j}} Z_{i}\right)-g\left(Z_{i}, \nabla_{Z_{j}} X-\nabla_{X} Z_{j}\right) \\
& =g\left(X, \nabla_{Z_{j}} Z_{i}\right)+g\left(Z_{i}, \nabla_{X} Z_{j}\right) .
\end{aligned}
$$

Summing up with $\left(L_{Z_{i}} \alpha_{j}\right)(X)$ and recalling that $\left[Z_{i}, Z_{j}\right]=0$, we get $g\left(X, \nabla_{Z_{j}} Z_{i}\right)=0$ for all $X$.

To prove the fourth property, let us consider the second fundamental form $B$ of an orbit $\tilde{M}$ of the Reeb action. Since the Reeb vector fields are tangent to the orbits of the Reeb action, we can choose $\left\{Z_{1}, Z_{2}\right\}$ (restricted to $\tilde{M}$ ) as a basis of the tangent space at every point of $\tilde{M}$. For a vector field $X$ on $M$ along $\tilde{M}$, let us denote by $X^{T}$ its tangential component. Then, by the third property, we have:

$$
B\left(Z_{i}, Z_{j}\right)=\nabla_{Z_{i}} Z_{j}-\left(\nabla_{Z_{i}} Z_{j}\right)^{T}=0
$$

Finally, if $g$ is associated, we want to prove that $\left[L_{Z_{i}} g\right](X, Y)=0$ for all $X, Y \in \Gamma(T M)$ if and only if $\left(L_{Z_{i}} \phi\right)(Y)=0$ for every $Y \in \Gamma(T M)$. This is clearly true for $Y=Z_{1}$ or $Z_{2}$, since in this case $\left[L_{Z_{i}} \phi\right]\left(Z_{j}\right)$ vanishes identically and $L_{Z_{i}} \alpha_{j}=0$ applied to a vector field $X$ implies $\left[L_{Z_{i}} g\right]\left(X, Z_{j}\right)=0$.

It remains to prove the assertion for $Y$ in $\operatorname{Ker} \alpha_{1} \cap \operatorname{Ker} \alpha_{2}$. In this case, for all vector fields $X, Y$, we have:

$$
\begin{aligned}
0 & =L_{Z_{i}}\left(d \alpha_{1}+d \alpha_{2}\right)(X, Y) \\
& =Z_{i} g(X, \phi Y)-\left(d \alpha_{1}+d \alpha_{2}\right)\left(L_{Z_{i}} X, Y\right)-\left(d \alpha_{1}+d \alpha_{2}\right)\left(X, L_{Z_{i}} Y\right) \\
& =\left[L_{Z_{i}} g\right](X, \phi Y)+g\left(X,\left[L_{Z_{i}} \phi\right](Y)\right)
\end{aligned}
$$

and this completes the proof since $\phi$ restricted to $\operatorname{Ker} \alpha_{1} \cap \operatorname{Ker} \alpha_{2}$ is an isomorphism.

Remark 14. Codimension two geodesible foliations of closed 4-manifolds have been studied by Cairns and Ghys in [9]. In particular, they have proven that, in this situation, there exists a metric on the manifold for which the leaves of the foliation are minimal and have same constant curvature $K=0,1,-1$. Our case belongs to what they have called parabolic case, that is $K=0$.

As for the metric contact structures (see [8] for example), by polarization one can show the existence of associated metrics, and in fact we have:

Proposition 15. For every contact pair $\left(\alpha_{1}, \alpha_{2}\right)$ on a manifold $M$, there exists an endomorphism $\phi$ of TM and a metric $g$ such that $\left(\alpha_{1}, \alpha_{2}, \phi, g\right)$ is a metric contact pair. Moreover $\phi$ can be chosen to be decomposable.

Proof. Take any Riemannian metric $k$. Since $d \alpha_{1}+d \alpha_{2}$ is symplectic on the subbundle $T \mathcal{G}_{1} \oplus_{\sim}$ $T \mathcal{G}_{2}$, then it can be polarized by using $k$ to obtain a metric $\tilde{g}$ and an almost complex structure $\tilde{\phi}$ 
compatible with $\tilde{g}$. Extending $\tilde{\phi}$ to be zero on the Reeb vector fields, we obtain $\phi$. Defining $g$ to be equal to $\tilde{g}$ on $T \mathcal{G}_{1} \oplus T \mathcal{G}_{2}$ and putting $g\left(X, Z_{i}\right)=\alpha_{i}(X)$, gives the desired metric.

To obtain a decomposable $\phi$, polarize $d \alpha_{i}$ on $T \mathcal{G}_{i}$ to obtain two metrics $\tilde{g}_{i}$ and two endomorphisms $\tilde{\phi}_{i}$ on $T \mathcal{G}_{i}(i=1,2)$ and then take the direct sums. Finally, extend them as before to obtain the desired tensors.

We end this section with two results concerning the structures induced on the leaves of the characteristic foliations:

Theorem 16. Let $M$ be a manifold endowed with a MCP $\left(\alpha_{1}, \alpha_{2}, \phi, g\right)$ and suppose that $\phi$ is decomposable. Then $\left(\alpha_{i}, \phi, g\right)$ induces a contact metric structure on the leaves of the characteristic foliation of $\alpha_{j}$ for $i \neq j, i, j=1,2$.

Proof. We will prove the assertion only for $\left(\alpha_{1}, \phi, g\right)$, the other case being completely similar. If $F$ is a leaf of the foliation $\mathcal{F}_{2}$, then $\left(\alpha_{1}, \phi\right)$ induces an almost contact structure on it and $\alpha_{1}$ is a contact form. Since $g$ is an associated metric with respect to $\left(\alpha_{1}, \alpha_{2}, \phi\right)$, by Definition 10, when restricted to vectors $X, Y$ which are tangent to $F$ we have:

$$
g(X, \phi Y)=d \alpha_{1}(X, Y), g\left(X, Z_{1}\right)=\alpha_{1}(X),
$$

showing that its restriction to $F$ is an associated metric with respect to the contact form induced by $\alpha_{1}$.

In a similar way, recalling that the characteristic foliation of $d \alpha_{i}$ for $i=1,2$ is given by ker $d \alpha_{i}$, we can prove:

Theorem 17. Let $M$ be a manifold endowed with a MCP $\left(\alpha_{1}, \alpha_{2}, \phi, g\right)$ and suppose that $\phi$ is decomposable. Then $\left(\alpha_{1}, \alpha_{2}, \phi, g\right)$ induces a metric contact pairs of type $(h, 0)$ on the leaves of the characteristic foliation of $d \alpha_{2}$ and one of type $(0, k)$ on the leaves of the characteristic foliation of $d \alpha_{1}$.

4.1. Orthogonal foliations. The following theorem explains the link between decomposability of $\phi$ and orthogonality of the characteristic foliations when the metric is an associated one:

Theorem 18. For a MCP $\left(\alpha_{1}, \alpha_{2}, \phi, g\right)$, the tensor $\phi$ is decomposable if and only if the foliations $\mathcal{F}_{1}, \mathcal{F}_{2}$ are orthogonal.

Proof. Suppose that $\phi\left(T \mathcal{F}_{i}\right) \subset T \mathcal{F}_{i}$ for $i=1,2$ and let $X \in \Gamma\left(T \mathcal{F}_{1}\right), Y \in \Gamma\left(T \mathcal{F}_{2}\right)$. Because $g$ is associated, by the choice of $X, Y$, we have:

$$
g(X, \phi Y)=d \alpha_{1}(X, Y)+d \alpha_{2}(X, Y)=0, g\left(X, Z_{1}\right)=\alpha_{1}(X)=0,
$$

which proves that $X$ is orthogonal to $T \mathcal{F}_{2}$ and then the two foliations are orthogonal.

Conversely, suppose that the two foliations are orthogonal. Then, for $X \in \Gamma\left(T \mathcal{F}_{1}\right)$ and every $Y \in \Gamma\left(T \mathcal{F}_{2}\right)$, we have

$$
g(Y, \phi X)=\left(d \alpha_{1}+d \alpha_{2}\right)(Y, X)=0
$$

which implies that $\phi X \in \Gamma\left(T \mathcal{F}_{2}^{\perp}\right)=\Gamma\left(T \mathcal{F}_{1}\right)$, that is $\phi$ is decomposable.

In the Example 8 the foliations are orthogonal with respect to the metric $g=\sum_{i=1}^{2}\left(d x_{i}^{2}+d y_{i}^{2}+\right.$ $\left.\alpha_{i}^{2}\right)$ which is compatible but not associated because $\phi$ is not decomposable.

Here is an example of MCP with decomposable $\phi$ on a nilpotent Lie group and its corresponding nilmanifolds: 
Example 19. Consider the simply connected nilpotent Lie group $G$ with structure equations:

$$
\begin{gathered}
d \omega_{1}=d \omega_{6}=0, d \omega_{2}=\omega_{5} \wedge \omega_{6}, \\
d \omega_{3}=\omega_{1} \wedge \omega_{4}, d \omega_{4}=\omega_{1} \wedge \omega_{5}, d \omega_{5}=\omega_{1} \wedge \omega_{6},
\end{gathered}
$$

where the $\omega_{i}$ 's form a basis for the cotangent space of $G$ at the identity.

The pair $\left(\omega_{2}, \omega_{3}\right)$ is a contact pair of type $(1,1)$ with Reeb vector fields $\left(X_{2}, X_{3}\right)$, the $X_{i}$ 's being dual to the $\omega_{i}$ 's. Now define $\phi$ to be zero on the Reeb vector fields and

$$
\phi\left(X_{5}\right)=-X_{6} \quad, \phi\left(X_{6}\right)=X_{5}, \phi\left(X_{1}\right)=-X_{4}, \phi\left(X_{4}\right)=X_{1} .
$$

Then $\phi$ is easy verified to be decomposable and the metric $g=\sum_{i=1}^{6} \omega_{i}^{2}$ is an associated metric with respect to $\left(\omega_{2}, \omega_{3}, \phi\right)$.

Since the structure constants of the group are rational, there exist lattices $\Gamma$ such that $G / \Gamma$ is compact. Since the MCP on $G$ is left invariant, it descends to all quotients $G / \Gamma$ and we obtain nilmanifolds carrying the same type of structure.

\section{FINAL COMMENTS}

Further metric properties of the contact pairs structures are studied in [5], where we prove, for example, that the characteristic foliations are minimal with respect to an associated metric.

In [4] we study the analog for a contact pair structure of the notion of normality for almost contact structures. We give there several constructions involving Boothby-Wang fibrations and flat bundles, which can be used to produce more examples of metric contact pairs.

\section{REFERENCES}

[1] G. Bande, Formes de contact généralisé, couples de contact et couples contacto-symplectiques, Thèse de Doctorat, Université de Haute Alsace, Mulhouse 2000.

[2] G. Bande, Couples contacto-symplectiques, Trans. Amer. Math. Soc. 355 (2003), 1699-1711.

[3] G. Bande, A. Hadjar, Contact Pairs, Tohoku Math. Journal 57 (2005), no. 2, 247-260.

[4] G. Bande, A. Hadjar, On normal contact pairs, arXiv:math.DG/0805.0193

[5] G. Bande, A. Hadjar, On the Riemannian geometry of contact pairs, in progress.

[6] G. Bande, P. Ghiggini, D. Kotschick, A stability theorem for contact and symplectic pairs, Int. Math. Res. Not., 68 (2004), 3673-3688.

[7] G. Bande, D. Kotschick, The Geometry of Symplectic pairs, Trans. Amer. Math. Soc. 358 (2006), no. 4, 16431655 .

[8] D. E. Blair, Riemannian geometry of contact and symplectic manifolds, Progress in Mathematics, vol. 203, Birkhäuser, 2002.

[9] G. Cairns, É. Ghys, Totally geodesic foliations on 4-manifolds, J. Differential Geom. 23 (1986), no. 3, 241-254

[10] D. Kotschick, S. Morita, Signatures of foliated surface bundles and the symplectomorphism groups of surfaces, Topology 44 (2005), no. 1, 131-149.

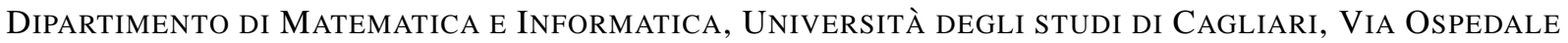
72, 09124 CAGLIARI, ITALY

E-mail address: gbande@unica. it

Laboratoire de Mathématiques, Informatique et Applications, Université de Haute Alsace 4 Rue de Frères Lumière, 68093 Mulhouse Cedex, France

E-mail address: Amine. Hadjar@uha.fr 